\title{
APLICAÇÃO DE PLANEJAMENTO SIMPLEX CENTROIDE PARA PADRONIZAÇÃO DE SOLUÇÕES EXTRATIVAS DAS FOLHAS DE ANACARDIUM OCCIDENTALE L.
}

\author{
L. K. S. CHAGAS ${ }^{1}$, R. T. M. RAMOS ${ }^{1,2}$, M. R. A. FERREIRA ${ }^{1,2}$, L.A.L. SOARES ${ }^{1,2}$ \\ ${ }^{1}$ Universidade Federal de Pernambuco, Laboratório de Farmacognosia, Departamento de \\ Ciências Farmacêuticas, \\ ${ }^{2}$ Universidade Federal de Pernambuco, Centro de Biociências, Programa de Pós-graduação \\ em Inovação Terapêutica.
}

E-mail para contato: laysa-karla@hotmail.com

RESUMO - As folhas de Anacardium occidentale L. (caju), possuem vasta utilização na medicina tradicional por apresentar atividade anti-inflamatória, antiulcerogênica, antimicrobiana e antifúngica. Essas atividades podem ser atribuidas à presença dos metabolitos secundários. Sabendo-se que a utilização do liquido extrator adequado no processo de extração permite obter maior rendimento desses compostos, o objetivo deste trabalho foi avaliar a influência dos solventes na determinação de resíduo seco através da ferramenta estatística simplex centroide. Os extratos foram preparados por turbólise na proporção droga: solvente $10 \%(\mathrm{~m} / \mathrm{v})$ utilizando os seguintes solventes: água, metanol, etanol e acetona; e suas misturas. O resíduo seco foi determinado em balança de infravermelho e os resultados foram avaliados pelo software Statistica, empregando o modelo matemático cúbico. A mistura binária dos solventes metanol e acetona $(50 \% ; v / v)$ foi a que apresentou maior teor de resíduo correspondendo a 4,92 $\pm 0,2011$. O planejamento simplex centroide mostra-se, portanto eficaz na avaliação da influência dos solventes no processo extrativo das folhas de caju.

Palavras-chave: Simplex centroide, Resíduo seco, Anacardium occidentale L.

ABSTRACT - The leaves of Anacardium occidentale L. (cashew) are widely used in traditional medicine due to several properties such as: antiemetic, treatment of gastritis, diarrhea, headaches, diabetes and hypertension. Their properties are attributed to the presence of secondary metabolites. Since the solvents play a pivotal role on the compound extraction, the purpose of this work was to evaluate the influence of the solvents on the total extraction performance by using a simplex centroid approach. The extracts were prepared by turbolysis in the ratio drug:solvent of $10 \%(\mathrm{~m} / \mathrm{v})$ using the following solvents: water, methanol, ethanol 
and acetone; and their mixtures. The extractives were determined by loss weight (infrared device) and the results were evaluated statically using cubic model. The binary mixture of the solvents methanol and acetone $(50 \% ; \mathrm{v} / \mathrm{v})$ showed the highest yield of extractives corresponding to $4.92 \pm 0.2011(\%$., w/w). The simplex centroid planning is therefore effective in evaluating the influence of solvents on the cashew extractive process.

Key words: Simplex centroid, Dry residue, Anacardium occidentale L.

\section{INTRODUÇÃO}

Anacardium occidentale L. (Anacardiaceae), é uma espécie encontrada nas regiões Norte, Nordeste, Centro-Oeste e Sudeste do Brasil (SILVA-LUZ; PIRANI, 2015). Bastante utilizada na medicina tradicional, ela compõe a Relação de Plantas Medicinais de interesse ao SUS (RENISUS) (BRASIL, 2009). Diversas atividades farmacológicas são atribuídas as folhas de A. occidentale tais como: anti-inflamatória (PAWAR et al., 2000), antiulcerogênica (KONAN; BACCHI, 2007), antimicrobiana e antifúngica (DAHAKE et al., 2009), devido à presença de fitoconstituintes originados do metabolismo secundário.A obtenção de constituintes químicos de interesse farmacológico depende de sistemas de solventes empregados nos métodos extrativos (DICIAULA et al., 2014). Para tanto, estudos preliminares devem ser realizados para possibilitar a análise da eficiência de extração, cuja medida relaciona o teor das substâncias marcadoras com o teor de sólidos que cada extrato apresenta. Portanto, é necessário realizar estudo acerca do resíduo seco, onde o solvente utilizado no processo extrativo influencia diretamente na sua composição qualitativa e quantitativa.

Nesse contexto, o planejamento de misturas Simplex Centroide vem sendo bastante empregado para avaliar a influência de cada componente presente na mistura sobre a resposta de variáveis dependentes (DICIAULA et al., 2014). Diante do explanado, o objetivo desse estudo foi de avaliar a influência dos sistemas de extração sobre o percentual de resíduos secos das folhas de $A$. occidentale.

\section{MATERIAIS E MÉTODOS}

As folhas de $A$. occidentale L. foram coletadas em Recife (PE) e identificadas no Instituto Agronômico de Pernambuco sob o número de tombamento 89991. Posteriormente, o material vegetal foi submetido à secagem em estufa (4 dias, $40^{\circ} \mathrm{C}$ ) e triturado em moinho de facas (Tipo Willye TE-680, Tecnal ${ }^{\circledR}$ ).

As soluções extrativas foram preparadas na proporção droga: solvente $10 \%(\mathrm{p} / \mathrm{v})$, utilizando-se extração por turbólise, com 4 ciclos de 30 segundos e intervalos de 5 minutos. Os solventes utilizados foram água, acetona, etanol e metanol, avaliados pelo planejamento Simplex Centroide, utilizando os solventes puros ou em misturas binárias, terciárias e quaternárias. As proporções de solventes para cada preparação se encontram na tabela 1.

A avaliação da resposta do método foi avaliada pela determinação de resíduo seco, realizada em balança de infravermelho $\left(\right.$ Marte $\left.^{\circledR}\right)$, utilizando $5 \mathrm{~mL}$ da solução extrativa, à temperatura de $130{ }^{\circ} \mathrm{C}$, até que não houvesse variação da massa em $0,02 \%$ durante 30 segundos.

As análises foram realizadas em triplicata e os resultados foram avaliados pelo software Statistica ${ }^{\circledR}$. 


\section{RESULTADOS E DISCUSSÃO}

A variação no teor de resíduos calculados a partir da massa resultante da secagem em balança de infravermelho permite verificar que a mudança na composição do solvente possui influência no processo extrativo, como mostra a tabela 1. Os resultados apresentados demonstram que os solventes puros apresentam baixo rendimento com exceção para o metanol, que apresentou o terceiro maior rendimento 4,31\%. A mistura binária acetona:metanol apresentou o melhor resultado de rendimento 4,92\% $\pm 0,2011$ $(4,09)$. A determinação do teor de resíduo seco torna-se importante para avaliar extratos que serão submetidos a processo de secagem, visto que possibilita obter previamente a medida do rendimento do material. Os resultados obtidos são importantes para estudos posteriores, onde pretende-se correlacionar a eficiência de extração com a medida de teores de substâncias de interesse.

Tabela 1 - Teor de resíduo seco para cada sistema de extração.

\begin{tabular}{cccccc}
\hline Extrato & $\mathbf{H}_{\mathbf{2}} \mathbf{O}$ & $\mathbf{M e O H}$ & $\mathbf{E t O H}$ & Acetona & \% Teor de resíduo \\
\hline $\mathbf{1}$ & 1 & 0 & 0 & 0 & $2,81 \pm 0,0500(1,78)$ \\
$\mathbf{2}$ & 0 & 1 & 0 & 0 & $4,31 \pm 0,0651(1,51)$ \\
$\mathbf{3}$ & 0 & 0 & 1 & 0 & $1,95 \pm 0,0808(4,14)$ \\
$\mathbf{4}$ & 0 & 0 & 0 & 1 & $1,74 \pm 0,0651(3,73)$ \\
$\mathbf{5}$ & 0,5 & 0,5 & 0 & 0 & $4,08 \pm 0,1436(3,52)$ \\
$\mathbf{6}$ & 0,5 & 0 & 0,5 & 0 & $4,12 \pm 0,0764(1,85)$ \\
$\mathbf{7}$ & 0,5 & 0 & 0 & 0,5 & $4,51 \pm 0,0693(1,54)$ \\
$\mathbf{8}$ & 0 & 0,5 & 0,5 & 0 & $4,06 \pm 0,0361(0,89)$ \\
$\mathbf{9}$ & 0 & 0,5 & 0 & 0,5 & $4,92 \pm 0,2011(4,09)$ \\
$\mathbf{1 0}$ & 0 & 0 & 0,5 & 0,5 & $2,08 \pm 0,2551(12,27)$ \\
$\mathbf{1 1}$ & 0,333 & 0,333 & 0,333 & 0 & $4,02 \pm 0,0252(0,63)$ \\
$\mathbf{1 2}$ & 0,333 & 0 & 0,333 & 0,333 & $4,04 \pm 0,1514(3,75)$ \\
$\mathbf{1 3}$ & 0,333 & 0,333 & 0 & 0,333 & $3,89 \pm 0,0681(1,75)$ \\
$\mathbf{1 4}$ & 0 & 0,333 & 0,333 & 0,333 & $4,08 \pm 0,0529(1,30)$ \\
$\mathbf{1 5}$ & 0,25 & 0,25 & 0,25 & 0,25 & $4,00 \pm 0,1380(3,45)$ \\
\hline
\end{tabular}

Onde: $\mathrm{H}_{2} \mathrm{O}$ : água; $\mathrm{MeOH}$ : metanol; EtOH: etanol. 1: solvente $100 \%$; 0,5: solvente $50 \%$; 0,333 : solvente $1 / 3 ; 0,25$ : solvente $25 \%$. Média $\pm \mathrm{DP}(\mathrm{CV})$

Com os resultados anteriores foi construído um modelo matemático para avaliar a influência dos solventes sobre o rendimento dos teores de resíduo seco. O modelo cúbico, que avalia as interações de três fatores, foi o que apresentou maior adequabilidade, devido ao valor de $R$ próximo a 1 . O gráfico de Paretos (Figura 1A) revelou a contribuição do diferentes solventes e respectivas misturas para a performance extrativa. Com apenas uma interação não significativa, a analise da superfície de resposta (Figura 1B), permite identificar as regiões com resposta de maior intensidade (em vermelho) correspondente à condição mais favorável à variável de resposta (teor de sólidos). Os achados estão de acordo com resultados descritos anteriormente, onde a mistura metanol:acetona foi a principal responsável pelo aumento no teor de sólidos de extratos vegetais. 
Figura 1 - Gráfico de Paretos (A) e Superfície de resposta (B) obtidos para o planejamento Simplex Centroide, referente ao resíduo seco com os solventes água, acetina e metanol.
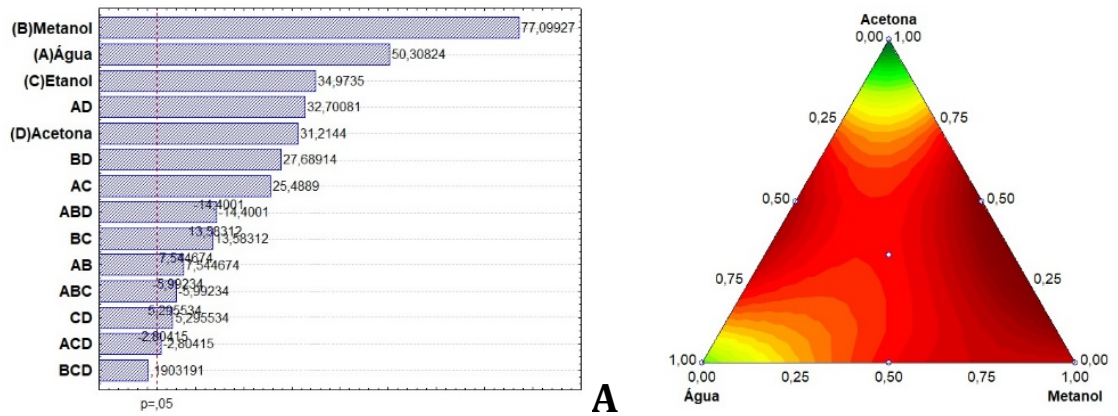

\section{CONCLUSÃO}

O modelo Simplex Centroide mostra-se eficaz para avaliar, identificar e quantificar a influência exercida por diferentes sistemas de solventes (puros e misturas) sobre o rendimento de sólidos em extratos de folhas de cajueiro obtidos por turbólise. A melhor condição foi alcançada com a mistura binária metanol:acetona. $\mathrm{O}$ incremento na performance extrativa de sólidos é passo tecnológico imprescindível para o desenvolvimento de processos de fabricação de formas sólidas. Tais resultados tornamse importantes, pois permite verificar o rendimento do material quando submetido a processos tecnológicos, como a obtenção de produtos secos.

\section{REFERÊNCIAS}

BRASIL. Relação de Plantas Medicinais de Interesse ao SUS. Brasília: Ministério da Saúde 2009.

DAHAKE, A.P.; JOSHI, V.D.; JOSHI, A. B. Antimicrobial Screening of Different Extract of Anacardium occidentale Linn. Leaves. Int. J. Chemtech. Res., v. 1, p. 856-858, 2009.

DICIAULA, M. C.; LOPES, G. C.; SCARMINIO, I. S.; MELLO, J. C. P. de M. Optimization of solvent mixtures for extraction from bark of Schinus terebinthifolius by a statistical mixture-design technique and development of a UV-Vis spectrophotometric method for analysis of total polyphenols in the extract.

Quim. Nova, v. 37, p. 159-163, 2014.

KONAN, N. A.; BACCHI, E. M. Antiulcerogenic effect and acute toxicity of a hydroethanolic extract from the cashew (Anacardium occidentale L.) leaves. J. Ethnopharmacol., v. 112, p. 237-243, 2007.

PAWAR, S. P.; SATHWANE, P. N.; METKAR, B. R.; PAL, S. C.; KASTURE, V. S.; KASTURE, S. B.; Anti-inflammatory and analgesic activity of Anacardium Occidentale Leaf Extracts. Anc Sci Life., v. 19 , p. $169-173,200$.

SILVA-LUZ, C. L.; PIRANI, J. R. Anacardiaceae in Lista de Espécies da Flora do Brasil. Jardim Botânico do Rio de Janeiro. Disponível em:<http://floradobrasil.jbrj.gov.br/jabot/floradobrasil/FB4381>, 2015.

\section{AGRADECIMENTOS}

Os integrantes agradecem a FACEPE (APQ-0493- 4.03/14) e ao CNPq (308386/20159). 